Göteborg-ITP-98-18

hep-th/9811145

November, 1998

\title{
Goldstone Tensor Modes
}

\author{
Tom Adawi, Martin Cederwall, Ulf Gran, \\ Bengt E.W. Nilsson and Behrooz Razaznejad
}

\author{
Institute for Theoretical Physics \\ Göteborg University and Chalmers University of Technology \\ S-412 96 Göteborg, Sweden
}

adawi, martin. cederwall, gran, bengt . nilsson, behrooz@fy . chalmers . se

\begin{abstract}
In the context of brane solutions of supergravity, we discuss a general method to introduce collective modes of any spin by exploiting a particular way of breaking symmetries. The method is applied to the D3, M2 and M5 branes and we derive explicit expressions for how the zero-modes enter the target space fields, verify normalisability in the transverse directions and derive the corresponding field equations on the brane. In particular, the method provides a clear understanding of scalar, spinor, and rank $r$ tensorial Goldstone modes, chiral as well as non-chiral, and how they arise from the gravity, Rarita-Schwinger, and rank $r+1$ Kalb-Ramond tensor gauge fields, respectively. Some additional observations concerning the chiral tensor modes on the M5 brane are discussed.
\end{abstract}


Adawi, Cederwall, Gran, Nilsson, Razaznejad: "Goldstone Tensor Modes" $\ldots \ldots \ldots \ldots \ldots 2$

\section{INTRODUCTION}

In theories with monopole or instanton solutions the study of moduli and collective coordinates has a long and interesting history. Quite generally two different kinds of moduli appear. E.g. in the context of the SU(2) gauge theory with a Higgs field one finds moduli describing the freedom to locate the monopole anywhere in space as well as one modulus stemming from the abelian gauge symmetry surviving the symmetry breaking. While moduli of the former kind are easily introduced by shifting the space coordinates, generating so called collective coordinates, the latter kind requires a more detailed analysis of the gauge theory itself. As described e.g. in [1], the fourth modulus of the BPS monopole arises from a special choice of gauge parameter corresponding to a large gauge transformation. In the following we will refer to both kinds of zero-modes as collective modes, while their constant part will be considered as moduli. Since we will be dealing with extended objects and their zero-modes, we do not find it fruitful to make a distinction between static and non-static configurations. In order to maintain the covariance of the dynamics of the zero-modes, which of course describe a field theory "on the brane", it is more fruitful to generalise the concept of motion on moduli space, relevant for point-like solitons, to variation of the collective coordinates with any of the (timelike or spacelike) longitudinal directions.

In the recent non-perturbative developments in string theory (see e.g. ref. [2]), these issues must be reexamined in the context of the $p_{-}$, $\mathrm{D}$-, and tensor branes appearing as solitonic solutions of various M-theory supergravity theories (for a review, see e.g. ref. [3]). The tensor brane M5, in particular, contains as collective modes self-dual tensor fields in six dimensions $[4,5,6]$, and will thus constitute a slightly more complicated but at the same time much more interesting example of these ideas. Common to all branes appearing as solutions to supergravity (in contrast to solitons in field theory without gravity) is the feature that all their collective modes are related to broken gauge symmetries and, as we will explain in detail below for the D3, M2 and M5 branes, these modes can be extracted from the target space gauge fields by making a judicious choice of the relevant gauge parameter.

Although the nature of the zero-modes discussed here has been known for some time and has been used in a number of applications, their explicit relationship to the brane solutions of supergravity has only been briefly touched upon $[7,8]$. We find it important that this situation is improved, so that the understanding of these aspects of string theory/M-theory solitons is put on a more equal footing to that of solitons in field theory.

The paper is organised as follows. In the next section we recapitulate the properties of $D=11$ supergravity [9] and type IIB supergravity in $D=10$ [10] that will be needed in subsequent sections. This section also sets the notation and introduces the various brane solutions on which we will focus our attention, namely the D3, M2 and M5 branes. Section

3 then describes the procedure which will tell us how the collective modes emerge from the 
target space fields. From this procedure it will also be clear in what sense the collective modes are related to broken symmetries. In particular, we will discover how self-dual gauge fields in six dimensions can arise from the three-form potential of $D=11$ supergravity. The order in which the collective modes are discussed is scalar, spinor, vector, and self-dual tensor. Section 3 ends by some comments on normalisability and other issues. In section 4 , some specific questions connected to the excitation of tensorial zero-modes are discussed, e.g. their electric charge. Section 5 contains a summary and some further comments.

\section{PRELiminaries}

The purpose of this section is to set the stage for the subsequent discussions of the M2 and M5 branes of $D=11$ supergravity and the D3 brane in type IIB $D=10$ supergravity. We will therefore start by reviewing these solutions and the field equations they solve. The conventions we use are listed in the appendix.

The bosonic action of eleven-dimensional supergravity is

$$
S=\int d^{11} x \sqrt{-g}\left(R-\frac{1}{48} H_{M N P Q} H^{M N P Q}\right)+\int \frac{1}{6} H \wedge H \wedge C,
$$

where the 4-form $H=d C$, which gives rise to the equations of motion

$$
R_{M N}-\frac{1}{2} g_{M N} R=\frac{1}{12} H_{M P Q R} H_{N}^{P Q R}-\frac{1}{96} g_{M N} H_{P Q R S} H^{P Q R S}
$$

and

$$
D^{M} H_{M N P Q}=\frac{\sqrt{|g|}}{2(4 !)^{2}} \varepsilon_{N P Q}{ }^{R_{1} \ldots R_{8}} H_{R_{1} \ldots R_{4}} H_{R_{5} \ldots R_{8}}
$$

or, equivalently, $d \star H=\frac{1}{2} H \wedge H$. It is often convenient to rewrite the first equation of motion as

$$
R_{M N}=\frac{1}{12} H_{M P Q R} H_{N}^{P Q R}-\frac{1}{144} g_{M N} H_{P Q R S} H^{P Q R S} .
$$

When we look at a specific brane solution we split the $M$ index into $(\mu, m)$, where $\mu$ denotes a direction on the brane and $m$ a direction transverse to the brane. For the extremal 2-brane [11] the solution [12] is

$$
\begin{aligned}
d s^{2} & =\Delta^{-\frac{2}{3}} \eta_{\mu \nu} d x^{\mu} d x^{\nu}+\Delta^{\frac{1}{3}} \delta_{p q} d y^{p} d y^{q}, \\
C & = \pm \frac{1}{3 !} \Delta^{-1} \varepsilon_{\mu \nu \rho} d x^{\mu} \wedge d x^{\nu} \wedge d x^{\rho}
\end{aligned}
$$


where

$$
\Delta=1+\left(\frac{R}{\rho}\right)^{6}
$$

and

$$
\rho=\sqrt{\delta_{m n} y^{m} y^{n}} .
$$

The corresponding extremal 5-brane solution [13] is

$$
\begin{aligned}
d s^{2} & =\Delta^{-\frac{1}{3}} \eta_{\mu \nu} d x^{\mu} d x^{\nu}+\Delta^{\frac{2}{3}} \delta_{m n} d y^{m} d y^{n}, \\
H & = \pm \frac{1}{4 !} \delta^{m n} \partial_{m} \Delta \varepsilon_{n p q r s} d y^{p} \wedge d y^{q} \wedge d y^{r} \wedge d y^{s}
\end{aligned}
$$

where $\Delta$ now is defined as

$$
\Delta=1+\left(\frac{R}{\rho}\right)^{3} .
$$

Both solutions are given in so called isotropic coordinates, where the isotropy groups $\mathrm{SO}(1, p)$ $\times \mathrm{SO}(11-p-1)$ are manifest and $\rho=0$ is the location of the horizon. The harmonic property of the $\Delta^{\prime}$ s, $\delta^{p q} \partial_{p} \partial_{q} \Delta=0$, is all that is needed to verify the solutions (2.5) and (2.8). The sign of the tensor field signifies positive or negative charge, i.e., a brane or an anti-brane. In the sequel, the positive sign will be chosen. The other sign will imply a switch of the chiralities of the zero-modes as will be clear in the following section. These comments also apply to the D3 extremal solution to which we now turn.

In the case of type IIB supergravity we give only the field equations to avoid the at this stage irrelevant discussion of actions for self-dual gauge fields. Here, and in the discussion of the zero-modes, we will need the field equations for the metric, the 2-form complex tensor potential $B$ and the 4 -form potential $C$ with self-dual field strength. We simplify the calculation by the initial observation that the scalar fields, taking values in the coset $\mathrm{SL}(2 ; \mathbb{R}) / \mathrm{U}(1)$, are constant in the D3 brane solution; the associated connections then vanish. The field strength of $C$ is $G=d C+i(\bar{B} \wedge H-B \wedge \bar{H})$, and, given that the scalars are constant, the complex 3-form field strength is $H=d B$.

The 2-form potential does not enter until we consider deformations of the D3 brane solution, so the relevant information in Einstein's equation reads

$$
R_{M N}=\frac{1}{96} G_{M P Q R S} G_{N}{ }^{P Q R S}+\text { other fields }
$$

while the equations for the tensors are

$$
G_{M N P Q R}=\star G_{M N P Q R}
$$

and

$$
d \star H+i G \wedge H=0
$$


(the last of these again makes use of the vanishing of the connections built from the scalars).

The D3 brane solution is now given by [14]

$$
\begin{aligned}
d s^{2}= & \Delta^{-\frac{1}{2}} \eta_{\mu \nu} d x^{\mu} d x^{\nu}+\Delta^{\frac{1}{2}} \delta_{m n} d y^{m} d y^{n} \\
G= & \pm \frac{1}{5 !}\left(\delta^{m n} \partial_{m} \Delta \varepsilon_{n p q r s t} d y^{p} \wedge d y^{q} \wedge d y^{r} \wedge d y^{s} \wedge d y^{t}\right. \\
& \left.+5 \partial_{m} \Delta^{-1} \varepsilon_{\mu \nu \rho \sigma} d y^{m} \wedge d x^{\mu} \wedge d x^{\nu} \wedge d x^{\rho} \wedge d x^{\sigma}\right)
\end{aligned}
$$

and $H=0$, where

$$
\Delta=1+\left(\frac{R}{\rho}\right)^{4}
$$

From now on all contractions are done with flat metric tensors which will not appear explicitly.

\section{ZERO-MODES}

The presence in spacetime of any object, like the extended ones discussed in the previous section, breaks some of the symmetries of the background. The breaking of these symmetries gives rise to Goldstone modes living on the branes. Since we are dealing with a supersymmetric theory, there will be both fermionic and bosonic Goldstone modes. Furthermore, since the branes discussed here leave half of the spacetime supersymmetry unbroken the Goldstone modes will fall into ordinary supermultiplets, for which the number of fermionic and bosonic modes are equal. The broken supersymmetries of the M2 brane solution give rise to eight Goldstone fermions, while the broken translational symmetry in the transverse directions, leads to eight Goldstone scalars. The M5 brane solution also breaks half of the supersymmetry, but it has only five transverse directions. The breaking of the translational symmetry in these transverse directions gives just five Goldstone scalars and thus there are three bosonic zero-modes missing. These bosonic zero-modes come from an (anti-)self-dual 3 -form and arise from breaking the gauge symmetry of the background 3 -form potential $C$ in exactly the same way as for the scalar and fermionic modes.

The viewpoint presented in the previous paragraph conforms with the standard picture of a BPS brane in flat space, breaking half of the rigid supersymmetries as well as the transverse translations. From the supergravity point of view, these are global symmetries of the asymptotic Minkowski region in the solutions of section 2. In the supergravity theory per se, without assuming a specific background, it is not meaningful to talk about global symmetries in this sense - all relevant symmetries are local (reparametrisations, local supersymmetry, tensor gauge symmetry). The discussion that follows identifies the parts of these symmetries 
that are relevant for the Goldstone mechanism in brane solutions and the properties of the solutions that are essential for the mechanism.

We discuss a method, which can be used to obtain all the zero-modes for both the M2 and M5 brane in $D=11$ and the D3 brane in $D=10$. The main idea is to start from global, or large, gauge transformations on the background fields. The precise sense in which the transformations are large is that the gauge parameters take different values in the asymptotic Minkowski region, $\rho>R$, and close to the horizon, $\rho<<R$. This step thus introduces the dependence on the moduli for all the fields that are affected by the transformation. By making these transformations local in the brane coordinates and requiring that the transformed fields satisfy the equations of motion, we obtain the transversal behavior of the target space fields and the equations of motion for the zero-modes, or collective coordinates as they will be called here. Now that we have put forward the general idea, we proceed to do the calculations.

\subsection{The Scalar Zero-modes}

We first consider the scalar zero-modes. Since these modes are related to the breaking of the translational symmetry in the transverse directions, the relevant symmetries are infinitesimal diffeomorphisms. Under such transformations the metric changes as

$$
h_{M N}=\delta g_{M N}=\mathscr{L}_{\varepsilon} g_{M N}=2 D_{(M} \varepsilon_{N)}
$$

where $M=(\mu, m)$ corresponds to the split into brane and transverse directions coordinatised by $x^{\mu}$ and $y^{m}$, respectively. We now want to compute the change that results from a coordinate transformation transverse to the brane with parameter $\varepsilon^{m}=\Delta^{s} \bar{\phi}^{m}$ (and $\varepsilon^{\mu}=0$ ), where $s$ is a parameter to be determined and $\bar{\phi}^{m}$ are constant moduli. In order to have an expression useful for all the cases under discussion we give the answer for general values of the dimensions $D$ of the target space and $d=p+1$ of the brane, and general parameters $\alpha$ and $\beta$ in the metric Ansatz

$$
d s^{2}=\Delta^{2 \alpha} d x^{2}+\Delta^{2 \beta} d y^{2} .
$$

This gives

$$
\begin{aligned}
& h_{\mu \nu}^{(m o d)}=2 \alpha \Delta^{s+2 \alpha-1}\left(\bar{\phi}^{p} \partial_{p} \Delta\right) \eta_{\mu \nu}, \\
& h_{\mu n}^{(m o d)}=0 \\
& h_{m n}^{(m o d)}=2 \Delta^{s+2 \beta-1}\left(s \bar{\phi}_{(m} \partial_{n)} \Delta+\beta \delta_{m n} \bar{\phi}^{p} \partial_{p} \Delta\right),
\end{aligned}
$$


where the superscript (mod) indicates that the gauge parameters $\bar{\phi}$ are constant. Note that if $\bar{\phi}^{m}$ had been functions on the brane at this stage, there would have been additional derivative terms in these expressions.

We now drop the bar on $\bar{\phi}^{m}$ and let them become functions on the brane: $\phi^{m}=$ $\phi^{m}(x)$. These functions are the zero-modes and will from now on be referred to as collective coordinates $(c c)$. The corresponding metric components are denoted $h_{M N}^{(c c)}$. They are still given by eq. (3.3), and they are no longer pure gauge transformations. This last fact is the reason why new physical modes appear in the theory. That they are exactly the zero-modes we are interested in will now be shown.

Having obtained the proper form of the metric Ansatz, we should now insert it into the Einstein equations. Since we will work only to linear order in the perturbations away from the brane solution, the following observation will be calculationally useful. Since both a given background and the background changed by a gauge transformation with constant $\bar{\phi}^{m}$ solve the field equations, only terms containing at least one $x$-derivative on $\phi^{m}(x)$ will survive. The variation of the Ricci tensor reads

$$
\delta R_{M N}^{(c c)}=-\frac{1}{2} \nabla^{Q} \nabla_{Q} h_{M N}^{(c c)}+\nabla_{(M} \nabla^{Q} h_{N) Q}^{(c c)}-\frac{1}{2} \nabla_{(M} \nabla_{N)} h_{Q}^{(c c) Q}+\text { non-derivative terms } \cdot(3 \cdot 4)
$$

Inserting the above metric Ansatz then gives

$$
\begin{aligned}
\delta R_{\mu \nu}^{(c c)}= & -\alpha \Delta^{s-1} \eta_{\mu \nu}\left(\partial^{\rho} \partial_{\rho} \phi^{m}\right) \partial_{m} \Delta \\
- & {[s+\alpha(d-2)+\beta(D-d)] \Delta^{s-1}\left(\partial_{\mu} \partial_{\nu} \phi^{m}\right) \partial_{m} \Delta, } \\
\delta R_{m n}^{(c c)}= & -\Delta^{s+2 \beta-2 \alpha-1}\left[s\left(\partial^{\mu} \partial_{\mu} \phi_{(m)}\right) \partial_{n)} \Delta+\beta \delta_{m n}\left(\partial^{\mu} \partial_{\mu} \phi^{p}\right) \partial_{p} \Delta\right], \\
\delta R_{\mu n}^{(c c)}= & \frac{s}{2} \Delta^{s-1} \partial_{\mu} \phi_{n} \partial^{m} \partial_{m} \Delta \\
+ & \left(\frac{s}{2}-\alpha+\beta-[s+\alpha(d-2)+\beta(D-d)]\right) \Delta^{s-1} \partial_{\mu} \phi^{p} \partial_{n} \partial_{p} \Delta \\
+ & \frac{1}{2} s[s+\alpha(d-2)+\beta(D-d)-1] \Delta^{s-2} \partial_{\mu} \phi_{n} \partial^{m} \Delta \partial_{m} \Delta \\
+ & \left(\alpha-\beta+\alpha \beta(D-2)-\frac{s}{2}+\left(1-\frac{s}{2}\right)[s+\alpha(d-2)+\beta(D-d)]\right) \\
& \quad \times \Delta^{s-2} \partial_{\mu} \phi_{m} \partial^{m} \Delta \partial_{n} \Delta .
\end{aligned}
$$

The variation of the Ricci tensor should now be equated to the variation of the RHS of (2.4). In fact, we get no contribution (with longitudinal derivatives) from the second term in the RHS of (2.4), which can be seen by considering the index structure ${ }^{\dagger}$. When computing $\delta T_{M N}^{(c c)}$ one immediately realises that $\phi^{m}$ will never appear acted on by two $x$-derivatives, and also, by considering the index structure, that both $\delta T_{\mu \nu}^{(c c)}$ and $\delta T_{m n}^{(c c)}$ are zero modulo

$\dagger$ Therefore, it is not essential to distinguish the RHS's of eqs. (2.2) and (2.4), and the latter will also be referred to as the stress tensor. 
$\phi^{m}$ terms without $x$-derivatives. Using $(3 \cdot 5), \delta R_{\mu \nu}^{(c c)}=0$ then implies

$$
\square \phi \equiv \partial^{\mu} \partial_{\mu} \phi^{n}=0
$$

and

$$
s+\alpha(d-2)+\beta(D-d)=0 .
$$

The first of these conditions also means that $\delta R_{m n}^{(c c)}=0$ is satisfied. It is interesting to note that for the branes we consider the parameters related to the metric Ansatz satisfy

$$
\alpha(d-2)+\beta(D-d)=1,
$$

implying that $s=-1$ in all cases. Eq. (3.8) is in fact a well-known condition which guarantees that no velocity dependent forces appear between branes when the brane action is expanded to lowest non-trivial order in the collective modes related to broken translations [15].

Before turning to the actual computation of the stress tensor variation we plug the condition (3.7) into the the variation of the Ricci tensor. This produces the much simpler expressions:

$$
\begin{aligned}
\delta R_{\mu \nu}^{(c c)} & =-\alpha \Delta^{s-1} \eta_{\mu \nu} \square \phi^{m} \partial_{m} \Delta, \\
\delta R_{m n}^{(c c)} & =-\Delta^{s+2 \beta-2 \alpha-1}\left(s \square \phi_{(m} \partial_{n)} \Delta+\beta \delta_{m n} \square \phi^{p} \partial_{p} \Delta\right), \\
\delta R_{\mu n}^{(c c)} & \left.=\Delta^{s-1}\left(\frac{s}{2} \partial^{\mu} \phi_{n} \partial^{\mu} \partial_{m} \Delta\left(\frac{s}{2}-\alpha+\beta\right) \partial_{\mu} \phi^{p}\right) \partial_{n} \partial_{p} \Delta\right) \\
& -\frac{1}{2} s \Delta^{s-2} \partial_{\mu} \phi_{n} \partial^{m} \Delta \partial_{m} \Delta \\
& +\left(\alpha-\beta+\alpha \beta(D-2)-\frac{s}{2}\right) \Delta^{s-2} \partial_{\mu} \phi_{m} \partial^{m} \Delta \partial_{n} \Delta .
\end{aligned}
$$

To verify the last of Einstein's equations we now derive the expression for the linearised stress tensor for the three different cases under discussion. We start by considering $D=11$ supergravity and its stress tensor given in equation (2.2). Both the metric and the 3 -form potential should now be varied under coordinate transformations. We will however not get any relevant contribution from the variation of the metric since there is no derivative acting on it. The variation of $C_{M N P}$ follows from

$$
\delta_{\varepsilon} C=\mathscr{L}_{\varepsilon} C+d \Lambda=\left(i_{\varepsilon} d+d i_{\varepsilon}\right) C+d \Lambda=i_{\varepsilon} H
$$

obtained by choosing the accompanying gauge transformation 2 -form parameter $\Lambda=-i_{\varepsilon} B$. Here $H$ is the background value which means that for the M5 brane solution the only non-zero components of $\delta C$ are

$$
\delta_{\varepsilon} C_{m n p}=-\Delta^{s} \phi^{q} \partial^{r} \Delta \varepsilon_{q m n p r} .
$$


As for the Ricci tensor only $\partial_{\mu} \phi^{m}$ terms need be kept in $\delta T_{M N}^{(c c)}$ when inserted into the field equations. Hence, for the M5 brane we get

$$
\begin{aligned}
& \delta T_{\mu \nu}^{(c c)}=\delta T_{m n}^{(c c)}=0, \\
& \delta T_{\mu n}^{(c c)}=\frac{1}{2} \Delta^{s-2}\left(\partial_{\mu} \phi_{n} \partial^{p} \Delta \partial_{p} \Delta-\partial_{\mu} \phi^{m} \partial_{m} \Delta \partial_{n} \Delta\right)
\end{aligned}
$$

and we actually get the same result for the M2 and D3 brane. In order to do the calculation for the M2 brane we use the dual formulation, where the stress tensor is given by

$$
T_{M N}=\frac{1}{6 \cdot 6 !} H_{M P_{1} \ldots P_{6}} H_{N}^{P_{1} \ldots P_{6}}-\frac{1}{12 \cdot 7 !} g_{M N} H_{P_{1} \ldots P_{7}} H^{P_{1} \ldots P_{7}}
$$

and the non-vanishing component of the variation is

$$
\delta_{\varepsilon} H_{\mu p_{1} \ldots p_{6}}=\partial_{\mu} \phi^{q} \varepsilon_{q p_{1} \ldots p_{6} r} \Delta^{s} \partial^{r} \Delta
$$

which we obtain as in (3.10).

Finally, in order to do the calculation for the D3 brane, we use the expression for the type IIB, $D=10$ stress tensor given in equation (2.10) and that the variation, obtained as before, is

$$
\delta_{\varepsilon} G_{\mu p_{1} \ldots p_{4}}=\partial_{\mu} \phi^{m} \partial^{n} \Delta \varepsilon_{n m p_{1} \ldots p_{4}} \Delta^{s}
$$

and

$$
\delta_{\varepsilon} G_{\mu_{1} \ldots \mu_{5}}=-5 \partial_{\left[\mu_{1}\right.} \phi^{m} \partial_{m} \Delta^{-1} \varepsilon_{\left.\mu_{2} \ldots \mu_{5}\right]} \Delta^{s} .
$$

However, the self-duality of $G$ requires that we also have

$$
\delta_{\varepsilon} G_{m n \mu \nu \rho}=-2 \partial^{\sigma} \phi_{[m} \partial_{n]} \Delta \varepsilon_{\sigma \mu \nu \rho} \Delta^{s-1}
$$

Using the various values for the parameters $D, d, \alpha, \beta$ for the three cases under study one concludes that the Einstein equations are all satisfied provided $\square \phi^{m}=0, s=-1$ and that the function $\Delta$ is harmonic in the transverse coordinates, i.e., that $\partial^{m} \partial_{m} \Delta=0$.

In order to check the normalisability of the bosonic zero-modes we integrate out the transversal dependence of the $R$ term in the action, thus obtaining an effective world-volume action for the zero-modes. We find that for all three branes the zero-modes are normalisable. From now on, the superscripts (mod) and $(c c)$ will be suppressed. 


\subsection{The Fermionic Zero-modes}

We now turn to the Goldstone fermions. The supersymmetry transformation in $D=11$ supergravity is given by*

$$
\delta \psi_{M}=\tilde{D}_{M} \zeta=D_{M} \zeta-\frac{1}{288}\left(\Gamma_{M}^{N P Q R}-8 \delta_{M}^{N} \Gamma^{P Q R}\right) \zeta H_{N P Q R}
$$

and in type IIB, $D=10$ supergravity, for vanishing $B$-field, by

$$
\delta \psi_{M}=\tilde{D}_{M} \zeta=D_{M} \zeta-\frac{i}{192} \Gamma^{N P Q R} \zeta G_{M N P Q R}
$$

which for convenience we write as

$$
\delta \psi=\tilde{D} \zeta=\partial \zeta+\omega \zeta+\chi \zeta
$$

the three terms denoting the derivative term, the spin connection term and the $H$ - or $G$-term, respectively. The general expressions for the spin connections are

$$
\begin{aligned}
\omega_{\mu} & =\frac{1}{4} \omega_{\mu A B} \Gamma^{A B}=\frac{1}{2} \alpha \Delta^{\alpha-\beta-1} \Gamma_{\mu} \Gamma^{m} \partial_{m} \Delta, \\
\omega_{m} & =\frac{1}{4} \omega_{m A B} \Gamma^{A B}=\frac{1}{2} \beta \Delta^{-1} \Gamma_{m}{ }^{n} \partial_{n} \Delta
\end{aligned}
$$

and we split the $\Gamma$ matrices according to

$$
\begin{aligned}
& \Gamma_{A}=\left(\gamma_{\alpha} \otimes \Sigma^{9}, \mathbb{1} \otimes \Sigma_{a}\right), \\
& \Gamma_{A}=\left(\gamma_{\alpha} \otimes \mathbb{1}, \gamma^{7} \otimes \Sigma_{a}\right), \\
& \Gamma_{A}=\left(\gamma_{\alpha} \otimes \mathbb{1}, \gamma^{5} \otimes \Sigma_{a}\right),
\end{aligned}
$$

for the M2, M5 and D3 brane, respectively. This split corresponds to splitting the group $\mathrm{SO}(1, D-1)$ into $\mathrm{SO}(1, d-1) \times \mathrm{SO}(D-d)$, i.e., into longitudinal and transverse directions.

We now start with the M5 brane and also take into account the $\chi$ terms. Using the M5 brane solution (2.8) and the split of the $\Gamma$ matrices, we obtain the following expressions for

* Since the background is purely bosonic, we drop all higher order terms in the gravitino field in transformations, covariant derivatives and equations of motion. 
the spin connection terms and the $H$-terms given in equation (3.20):

$$
\begin{aligned}
\omega_{\mu} \zeta & =-\frac{\theta}{12} \Delta^{-\frac{3}{2}} \partial_{m} \Delta \gamma_{\bar{\mu}} \Sigma^{\bar{m}} \zeta, \\
\omega_{m} \zeta & =\frac{1}{6} \Delta^{-1} \partial_{n} \Delta \Sigma_{\bar{m}}{ }^{\bar{n}} \zeta \\
\chi_{\mu} \zeta & =-\frac{1}{12} \Delta^{-\frac{3}{2}} \partial_{m} \Delta \gamma_{\bar{\mu}} \Sigma^{\bar{m}} \zeta, \\
\chi_{m} \zeta & =-\frac{\theta}{12} \Delta^{-1} \partial_{m} \Delta \zeta+\frac{\theta}{6} \Delta^{-1} \partial_{n} \Delta \Sigma_{\bar{m}}{ }^{\bar{n}} \zeta,
\end{aligned}
$$

where $\theta= \pm 1$ is the 6 -dimensional chirality of $\zeta\left(\gamma_{7}\right.$ has simply been replaced by its eigenvalue $\theta$ ). Overlined indices are inertial indices - we prefer to use inertial $\Gamma$-matrices in order to manifest explicitly all radial dependence. A gauge transformation with $\zeta=\Delta^{k} \lambda$, where $\lambda$ is a constant spinor, gives

$$
\begin{aligned}
\delta \psi_{\mu} & =-\frac{\theta+1}{12} \Delta^{k-\frac{3}{2}} \Sigma^{\bar{m}} \partial_{m} \Delta \gamma_{\bar{\mu}} \lambda, \\
\delta \psi_{m} & =\left(k-\frac{\theta}{12}\right) \Delta^{k-1} \partial_{m} \Delta \lambda+\frac{\theta+1}{6} \Delta^{k-1} \partial_{n} \Delta \Sigma_{\bar{m}}{ }^{\bar{n}} \lambda
\end{aligned}
$$

From the equations above we see that the surviving supersymmetry, obeying the Killing spinor equation $\delta \psi=0$, has $\theta=-1$ and $k=-\frac{1}{12}$. To obtain the Goldstone fermions we let the gauge parameter $\lambda$ be $x$-dependent and require $\psi$, still given by eq. (3.24), to satisfy the equation of motion

$$
T_{M} \equiv \Gamma^{N} T_{M N}=0,
$$

where $T_{M N}=2 \tilde{D}_{[M} \psi_{N]}$ is the field strength of $\psi$. Eq. (3.25) is equivalent to the usual Rarita-Schwinger equation $\Gamma^{M N P} \tilde{D}_{N} \psi_{P}=0$, but easier to handle. Since by performing a global gauge transformation on a solution of the equations of motion we just obtain another solution, only the $\partial_{\mu} \lambda$-terms in the field strength have to be considered. They are

$$
\begin{aligned}
\left.T_{\mu \nu}\right|_{\partial \lambda} & =\frac{\theta+1}{6} \Delta^{k-\frac{3}{2}} \partial_{m} \Delta \gamma_{[\bar{\mu}} \Sigma^{\bar{m}} \partial_{\nu]} \lambda, \\
\left.T_{\mu m}\right|_{\partial \lambda} & =\Delta^{k-1} \partial_{n} \Delta\left[\left(k-\frac{\theta}{12}\right) \delta_{\bar{m}}^{\bar{n}}+\frac{\theta+1}{6} \Sigma_{\bar{m}}^{\bar{n}}\right] \partial_{\mu} \lambda, \\
\left.T_{m n}\right|_{\partial \lambda} & =0 .
\end{aligned}
$$

The $m$-component of the linearised equation of motion (3.25) becomes

$$
\left.T_{m}\right|_{\partial \lambda}=-\Delta^{k-\frac{5}{6}} \partial_{n} \Delta\left[\left(k-\frac{\theta}{12}\right) \delta_{\bar{m}}^{\bar{n}}+\frac{\theta+1}{6} \Sigma_{\bar{m}}^{\bar{n}}\right] \gamma^{\bar{\mu}} \partial_{\mu} \lambda=0
$$

This equation gives immediately the Dirac equation, $\gamma^{\mu} \partial_{\mu} \lambda=0$, for any mode except the one corresponding to the unbroken supersymmetry. Using the Dirac equation the other 
component of the trace can be written as

$$
\left.T_{\mu}\right|_{\partial \lambda}=\Delta^{k-\frac{4}{3}} \partial_{m} \Delta \Sigma^{\bar{m}} \partial_{\mu} \lambda\left(k+\frac{\theta}{4}+\frac{1}{3}\right)=0 .
$$

Assuming that the broken supersymmetry must have the opposite chirality compared to the unbroken one (as must be case if the zero-mode is part of a $D=6$ tensor multiplet), we get from the equation above that $k=-\frac{7}{12}$. Thus, we have obtained the equation of motion and the transversal behaviour of the Goldstone fermion living on the M5 brane. Performing the same calculations for the M2 brane we obtain $\theta=+1$ and $k=-\frac{1}{6}$ for the unbroken supersymmetry and $\theta=-1$ and $k=-\frac{4}{6}$ for the broken one. The only difference in this calculation is that $\theta$ now denotes the 8-dimensional chirality of $\zeta$.

In order to do the same calculation for the D3 brane, we take $\zeta$ to have positive 10dimensional chirality which implies that the 4 and 6 -dimensional chiralities of $\zeta$ must be the same, denoted by $\theta$. With the convention that

$$
\gamma^{\mu \nu \rho \sigma}=i \varepsilon^{\mu \nu \rho \sigma} \gamma^{5}
$$

we find that $\theta=-1$ and $k=-\frac{1}{8}$ for the unbroken supersymmetry while $\theta=+1$ and $k=-\frac{5}{8}$ for the broken one.

To check the obtained values of $k$ we now examine the supersymmetry algebra, which we schematically write as

$$
\left[\zeta Q, \zeta^{\prime} Q\right]=\zeta \Gamma^{M} \zeta^{\prime} \mathscr{L}_{M}+\ldots=\varepsilon^{M} \mathscr{L}_{M}+\ldots
$$

(only the diffeomorphisms are written out in the RHS). The unbroken supersymmetry must generate translations in the longitudinal directions that have no $y$-dependence, giving that

$$
\zeta \Gamma^{\mu} \zeta^{\prime}=\left(\Delta^{k} \lambda\right)\left(\Delta^{-\alpha} \Gamma^{\bar{\mu}}\right)\left(\Delta^{k} \lambda^{\prime}\right)
$$

must be independent of $y$. We thus get $k=\frac{\alpha}{2}$ for the unbroken supersymmetry in agreement with our previous results. In order to generate a translation in the transverse directions, which we have seen must behave as $\varepsilon^{m}=\Delta^{-1} \phi^{m}$, we must commute a broken and an unbroken supersymmetry generator, giving

$$
\zeta \Gamma^{m} \zeta^{\prime}=\left(\Delta^{k} \lambda\right)\left(\Delta^{-\beta} \Gamma^{\bar{m}}\right)\left(\Delta^{k^{\prime}} \lambda^{\prime}\right)=\Delta^{-1} \phi^{m}
$$


where $k^{\prime}$ denotes the exponent for the broken supersymmetry. We get $k^{\prime}=\beta-k-1$, which also agrees with our previous results. Inclusion of the tensor gauge transformations of the following two subsections in the RHS of eq. (3.30), thus relating the transverse behaviour of the fermionic and tensorial modes, gives the same result.

In all three cases under consideration, we find that the fermion zero-modes are normalisable in the transverse directions, so that the effective action reduces to that of a chiral spinor $^{\dagger}$ in the longitudinal directions. We also note that the assumption concerning the chirality of the fermion zero-modes made above is unnecessary, since the field strength obtained from the other chirality, i.e., the conserved supersymmetry, is identically zero.

\subsection{The Vector Zero-modes}

We will now see the first example of how broken tensor gauge symmetries give rise to zeromodes. We know that there is a vector field living on the D3 brane and the question is now how to interpret this field as arising from broken gauge symmetries. Since a vector zero-mode comes from a broken vector, or 1-form, gauge parameter we must have a corresponding 2form potential and 3-form field strength in which the zero-modes live. Of course, $D=10$ supergravity contains a 3 -form field strength $H$ corresponding to the 2 -form potential $B$ [10]. It is important to note that these supergravity fields are complex. We now make a gauge transformation $\delta B=d \Lambda$ and make the Ansatz $\Lambda=\Delta^{k} A$, where $A$ is a constant 1-form which lies in the longitudinal directions. The reason why we take $A$ to lie in the longitudinal directions is of course that we want to be able to integrate out the transversal dependence, thus obtaining an effective vector theory on the brane world-volume. We get

$$
\delta B=d \Delta^{k} \wedge A
$$

We now let $A$ become $x$-dependent, which means that it is no longer a pure gauge transformation. By computing $\delta H$ and solving the equations of motion for the variation we will get the equations of motion and the transversal behaviour for the zero-modes. We find

$$
\delta H=d(\delta B)=-d \Delta^{k} \wedge F
$$

$F$ being the complex 2-form field strength on the brane, $F=d A$, and when we look at the equations of motion [10]

$$
d \star H+i G \wedge H=0
$$

\footnotetext{
${ }^{\dagger}$ For the membrane, chirality refers to the internal Spin(8) indices.
} 
Adawi, Cederwall, Gran, Nilsson, Razaznejad: "Goldstone Tensor Modes" .......... 14

and use $G$ from (2.12), we get

$$
\Delta d \star_{x} F \wedge \star_{y} d \Delta+\left(i F-k \star_{x} F\right) \wedge d \Delta \wedge \star_{y} d \Delta=0
$$

The notation $\star_{x}$ and $\star_{y}$ implies dualisation with flat metrics $\eta_{\mu \nu}$ and $\delta_{m n}$. In the case of the longitudinal 2-form $F$, this makes no difference from using the restriction of the actual metric.

We now consider the two four-dimensional duality components of $F$, fulfilling $\star_{x} F=$ $\pm i F$, separately. The two terms in eq. (3.36) have different index structure and vanish independently. The first term gives that $d F=0$, which together with the relation $\star_{x} F= \pm i F$ is the equation of motion for $F$ and hence for the zero-modes. The second term determines the value of $k$ and we have $k=1$ for the positive sign and $k=-1$ for the negative sign. Each duality component of $F$ contributes with two modes and naïvely we thus have twice the number of modes we wanted. However, by requiring normalisability the positive sign is forbidden and we are left with the desired number of zero-modes.

The (anti-)self-duality of $F$ reflects the self-duality property of the D3 brane itself, and is connected to the fact that it forms a singlet under the $\operatorname{SL}(2 ; \mathbb{Z})$ symmetry of type IIB. Since we use a formalism for the supergravity where this symmetry is manifest, we do not obtain the real vector potential of the Born-Infeld theory for the zero-modes, but instead a complex one satisfying a complex self-duality. This ties up naturally with the work of ref. [16], where the D3 brane was given an $\mathrm{SL}(2 ; \mathbb{Z})$-covariant formulation. The fields used there are identical to the ones obtained here.

\subsection{The Tensor Zero-modes}

Finally, we discuss the tensor modes living on the M5 brane. As mentioned in the beginning of this section, these modes are related to the breaking of the gauge symmetry of the background 3-form potential $C$. Hence, we consider an infinitesimal gauge transformation $\delta C=d \Lambda$ and make the Ansatz $\Lambda=\Delta^{k} A$, where $A$ is a constant 2-form which lies in the longitudinal directions. Along the lines of the previous discussions we let $A$ become $x$-dependent and use the Ansatz to obtain the following expression for the variation of the $H$-field

$$
h=\delta H=F \wedge d \Delta^{k},
$$

where $F=d A$. We now require this expression to satisfy the equation of motion (2.3) to linear order,

$$
d \star h-H \wedge h=0 .
$$


We thus obtain

$$
\Delta d \star_{x} F \wedge \star_{y} d \Delta-\left(k \star_{x} F-F\right) \wedge d \Delta \wedge \star_{y} d \Delta=0 .
$$

We now consider the two 6-dimensional duality components of $F$ (fulfilling ${ }_{{ }_{x}} F= \pm F$ ) separately. The first term in the equation above gives immediately that $d F=0$, which together with the relation $\star_{x} F= \pm F$ is the equation of motion for the $F$-field. The second term determines the constant $k$ and we have $k=-1$ for the anti-self-dual part and $k=1$ for the self-dual part. Each duality component of $F$ contributes with three modes and as in the case of the vector zero-mode we naïvely have twice as many zero-modes as we wanted. Again, normalisability forbids one part of $F$, in this case the self-dual part. Of course, the quadratic $H$ term in the action vanishes when an (anti-)self-dual field is inserted. This may be seen as a cancellation between the kinetic and potential parts of $L=K-V$. Demanding that the energy $E=K+V$ is finite per unit brane volume amounts to the naïve transverse normalisability condition on the mode function. It is also noteworthy that the combination entering the action, namely the product of the two chiralities, is not normalisable, so the self-dual component can not serve as an auxiliary field. We finally note that the transversal behaviour for the normalisable tensor zero-modes given in [8] does not seem correct.

\subsection{Summary of Normalisable Zero-modes}

We end this section by noting that we have obtained the transverse behaviour and the equations of motion for all the zero-modes living on the M2, M5 and D3 brane by following a common procedure. The normalisable zero-modes we found are most conveniently summarised in terms of the gauge parameters containing the moduli:

$$
\begin{array}{llllll} 
& & & \text { M2 } & \multicolumn{1}{c}{\text { M5 }} & \multicolumn{1}{c}{\text { D3 }} \\
\text { Diffeomorphisms: } & \varepsilon^{m}=\Delta^{-1} \phi^{m} & \Delta^{-1} \phi^{m} & \Delta^{-1} \phi^{m} \\
\text { Local supersymmetry: } & \zeta=\Delta^{-\frac{2}{3}} \lambda_{-} & \Delta^{-\frac{7}{12}} \lambda_{+} & \Delta^{-\frac{5}{8}} \lambda_{+} \\
\text {Tensor gauge symmetry: } & \Lambda= & & \Delta^{-1} A & \Delta^{-1} A \\
& \Lambda & & (\star F=F) & (i \star F=F)
\end{array}
$$

As already mentioned, it is straightforward to check explicitly that the collective coordinates, i.e., the fields on the branes, form multiplets under the unbroken supersymmetries generated by the Killing spinors of section 3.2, thus providing a further check that the modes in eq. (3.40) are correct.

We note that the two distinguished fermionic modes, namely the Killing spinor and the fermionic zero-mode, in addition to having opposite chiralities, carry different dependence on the transverse coordinates. It does not make sense, except asymptotically, to think of 
Adawi, Cederwall, Gran, Nilsson, Razaznejad: "Goldstone Tensor Modes" ......... 16

the two as making up a non-chiral spinor of broken + unbroken supersymmetry. The zeromodes are in fact just one out of an infinite number of supersymmetries broken by the brane solution.

The vector modes of the D3 brane and the tensor modes of the M5 brane follow a very similar pattern, where only one out of two duality components is allowed by normalisability (finite energy condition).

In the Ansätze used for finding the transverse behaviour of the collective coordinates, we have used the constant mode on the transverse spheres. In principle, a general Ansatz would contain also higher Kaluza-Klein modes, but that discussion was postponed for simplicity. It is straightforward, and we will not go into details here, to show that such higher modes will not contain zero-modes - they will lead to massive fields on the brane. Another assumption, motivated by the knowledge of the presence of vector or tensor modes, was the index structure of the tensor gauge transformations, i.e., that the gauge parameters should carry only longitudinal indices (and corresponding statements for the other modes). This assumption is also straightforwardly verified by considering a more general Ansatz - the selfduality is the essential property that enables us to obtain massless modes by a cancellation between the two terms, originating from the kinetic term and Chern-Simons term, in the tensor equations of motion $(3 \cdot 35)$ or $(3 \cdot 38)$.

\section{Charges of the Excited M5 Brane}

The zero-modes of the M5 brane form a multiplet under the unbroken supersymmetry generated by the Killing spinors that make eq. (3.24) vanish, i.e., under a 6 -dimensional $(2,0)$ supersymmetry algebra. The amount of supersymmetry of the solution equals half the number of Killing spinors of the asymptotic Minkowski space - the extremal brane solutions are half-supersymmetric BPS configurations. The central charge in the 11-dimensional supersymmetry algebra is a 5 -form, the magnetic charge of the M5 brane. This algebra is obtained by anticommuting the unbroken and broken supersymmetries of section 3.2 in the asymptotic Minkowski region and using the background value of the tensor field.

Here we want to discuss briefly the corresponding situation when the tensorial zeromodes are excited. The first thing to observe is that once the tensor mode is turned on, the M5 brane no longer carries only magnetic charge, but also electric. The electric charge is measured by the flow out of a closed 7-surface. If this hypersurface is the contractible boundary of an 8-volume $\mathscr{M}_{8}$, the electric charge vanishes:

$$
e=\int_{\partial_{\mathscr{M}_{8}}}\left(* H-\frac{1}{2} H \wedge C\right)=\int_{\mathscr{M}_{8}}\left(d * H-\frac{1}{2} H \wedge H\right)=0
$$


due to the equation of motion for $H_{(4)}$ (or equivalently, the Bianchi identity for $H_{(7)}$ ). If however $\mathscr{M}_{8}$ intersects with the horizon $\Sigma_{5}$ of the M5 brane, $\rho=0$, its boundary can not be freely contracted. Without deforming the intersection with $\Sigma_{5}$, one may contract to the product of the intersection and a small 5-ball centered around each point in the intersection: $\mathscr{M}_{8} \rightarrow \mathscr{M}_{8}^{\prime}=\left(\mathscr{M}_{8} \cap \Sigma_{5}\right) \times B^{5}(\varepsilon)$. The electric charge may now be calculated as the integral over $\partial \mathscr{M}_{8}^{\prime}$. Using the explicit solution of section $3 \cdot 4$, the only contribution comes from the first term in the integral in eq. (4.1) over $\left(\mathscr{M}_{8} \cap \Sigma_{5}\right) \times S^{4}(\varepsilon)$, and the result is

$$
e=q \int_{\mathscr{M}_{8} \cap \Sigma_{5}} F \text {, }
$$

where $q=\int_{S^{4}} H=8 \pi^{2} R^{3}$ is the magnetic charge of the 5-brane. The corresponding statement is true for the D3 brane, where an excitation of the Born-Infeld field carries charge with respect to the NS-NS and RR 2 -form potentials. A $\mathrm{D}_{p}$-brane generically intersects the 8-dimensional hypersurface defining the charge in a $(p-1)$-dimensional hypersurface, and the charge will have to be expressed as an integral $e \sim \int_{\mathscr{M}_{8} \cap \Sigma_{p}} F_{(p-1)}$. The fields $F_{(p-1)}$ are those naturally obtained in the formalism of ref. [16].

The expression for the electric charge (4.2) is a topological quantity on the brane. The 3-dimensional manifold may enclose stringlike objects, the self-dual string solitons of ref. [17], and the integral measures the string charge.

The electric charge of the branes with excited tensors/vectors parallels the situation for monopoles in field theory, where momentum in the fourth direction of the moduli space is identified as electric charge. The analogy here is the field strength on the brane. As for the monopoles, the electric charge in equations (4.1) and (4.2) is the classical expression-charge quantisation is not seen at this level.

It is known from existing formulations of the dynamics of branes with vector or tensor degrees of freedom $[18,19,16,5,6]$ that the brane actions are $\kappa$-symmetric, i.e., there exists a projection of the target space spinor coordinates that are purely gauge degrees of freedom. For the M5 brane this projection looks like [5] $P=\frac{1}{2}(\mathbb{1} \pm \Gamma)$, where (barring numerical factors)

$$
\Gamma \sim \frac{\varepsilon^{\mu_{1} \ldots \mu_{6}}}{\sqrt{-g}}\left(\Gamma_{\mu_{1} \ldots \mu_{6}}+F_{\mu_{1} \mu_{2} \mu_{3}} \Gamma_{\mu_{4} \mu_{5} \mu_{6}}\right)
$$

The first term defines chirality with respect to the spinor decomposition (3.22) and are related to the magnetic charge of the M5 brane, or, more generally, to (electric) charge with respect to a potential $C_{(p+1)}$, while the second term is related to the electric charge (4.2), and corresponds to a 2-form extension in the 11-dimensional supersymmetry algebra. In reference [20], extensions of the 11-dimensional supersymmetry algebra were analysed from the point of view of the M5 brane, and it was shown how the 2-form extension is related 
Adawi, Cederwall, Gran, Nilsson, Razaznejad: "Goldstone Tensor Modes" .......... 18

to the self-dual tensor. Here, we have added a more direct ingredient to this interpretation, namely the resulting charges obtained by using the explicit form of the tensor field $H$ when the self-dual tensor is excited.

The above analysis is presented here only to linear order in $F$, and we refer to a forthcoming publication [21] for the case of finite field strengths.

\section{Discussion}

We have presented a principle, intimately connected to gauge symmetries, by which the zero-modes around a brane solution in supergravity can be found and explicitly constructed. The procedure has been carried through in detail for the membrane and 5-brane in eleven dimensions, as well as for the D3 brane of type IIB in ten dimensions.

The nature of the construction emphasises the way the zero-modes arise as Goldstone modes of a broken global symmetry. We would like to stress again that the relevant symmetries of the (supergravity) theories in question are gauge symmetries: reparametrisations, local supersymmetry and gauge symmetry of the tensor fields. The zero-modes building the supersymmetric field theories on the branes arise as Goldstone modes for breaking of certain modes of these symmetries which are large gauge transformations, and are as such global rather than local symmetries in the given backgrounds. The property of the brane configurations permitting such transformations is the fact that they contain different unconnected asymptotic regions (the asymptotic Minkowski region far from the brane and the near-horizon AdS region), and the gauge parameters may take different values in the two regions. It is easily seen that the relevant modes have exactly this property. This hinges on the fact that the transverse behaviour of the collective coordinates carry negative powers of the harmonic functions $\Delta$, so that the difference from horizon to infinity is well defined and finite: $\left(\lim _{\rho \rightarrow \infty}-\lim _{\rho \rightarrow 0}\right) \Delta^{-p}=1$.

The tensor or vector modes have sometimes been considered as more mysterious than the other ones, especially concerning their Goldstone properties [22] (maybe as a result of picturing the Goldstone mechanism as connected to asymptotic isometries instead of large gauge transformations). The present analysis, in contrast, treats all modes on equal footing. We should maybe remark that, although the popular picture of e.g. the scalar modes as Goldstone modes for the breaking of translational symmetry is appealing, the notion of translational symmetry in a gravity theory is somewhat suspect. The relevant symmetry is a large reparametrisation, having different asymptotic values far from the brane and near the horizon. It does not correspond to a rigid shift of the transverse coordinates in the solutions of section 2 . 
It is satisfying to see that the tensor or vector fields that arise are exactly those that appeared in refs. $[16,5]$, where they arose as fields on the branes, having the natural couplings to background fields and reflecting the symmetries of the background theory.

The entire construction is performed at linear level around infinite flat branes, which means that some aspects connected to non-linearities-Born-Infeld dynamics of D-branes and the corresponding non-linear dynamics in eleven dimensions - are not seen. Although these represent higher-derivative terms in an action, and therefore seem irrelevant for a discussion of the low-energy behaviour, these excitations are such that the BPS property is exactly preserved. States found in a quantum field theoretic treatment of the full nonlinear theory are therefore reliable; the concept of low energy should really be replaced by preservation of the BPS property. We have recently noted that it is possible to solve the coupled system of equations of motion for the gravity and tensor field to all orders, which results in a brane solution with (constant) finite tensor field strength. This will be reported in ref. [21].

\section{Appendix A: Conventions}

We use the conventions that

$$
\eta_{\mu \nu}=(-1+1+1 \ldots+1)
$$

and that the Levi-Civita tensor density $\varepsilon$ with downstairs indices is defined to be +1 .

Our convention for dualisation of a $p$-form in $D$ dimensions is

$$
\star\left(d x^{M_{1}} \wedge d x^{M_{2}} \wedge \ldots \wedge d x^{M_{p}}\right)=\frac{\sqrt{|g|}}{(D-p) !} \varepsilon^{M_{1} \ldots M_{p}} M_{p+1} \ldots M_{D} d x^{M_{p+1}} \wedge \ldots \wedge d x^{M_{D}}
$$

which translated to acting on the tensor components reads

$$
(\star \Omega)_{M_{1} \ldots M_{D-p}}=\frac{\sqrt{|g|}}{p !} \Omega_{N_{1} \ldots N_{p}} \varepsilon^{N_{1} \ldots N_{p}}{ }_{M_{1} \ldots M_{D-p}},
$$

with the convention that

$$
\Omega_{(p)}=\frac{1}{p !} d x^{M_{1}} \wedge d x^{M_{2}} \wedge \ldots \wedge d x^{M_{p}} \Omega_{M_{1} \ldots M_{p}} .
$$


Acknowledgements: The authors would like to thank Måns Henningson for discussions.

\section{REFERENCES}

[1] R. Rajaraman, "Solitons and instantons", North-Holland, 1982;

J.A. Harvey, "Magnetic monopoles, duality and supersymmetry", hep-th/9603086.

[2] E. Witten, "String theory dynamics in various dimensions",

Nucl. Phys. B443 (1995) 85 hep-th/9503124;

C.M. Hull and P.K. Townsend, "Unity of superstring dualities",

Nucl. Phys. B438 (1995) 109 hep-th/9410167;

J.H. Schwarz, "The power of M theory", Phys. Lett. B367 (1996) 97 hep-th/9510086;

A. Sen, "Unification of string dualities", Nucl. Phys. Proc. Suppl. 58 (1997) 5 hep-th/960917];

P.K. Townsend, "Four lectures on M-theory", hep-th/9612121.

[3] M.J. Duff, "Supermembranes", hep-th/9611203.

[4] E. Witten, "Five-brane effective action in M-theory", J. Geom. Phys. 22 (1997) 103 hep-th/9610234;

M. Aganagic, J. Park, C. Popescu and J.H. Schwarz,

"World-volume action of the M theory five-brane", Nucl. Phys. B496 (1997) 191 hep-th/9701166;

T. Adawi, M. Cederwall, U. Gran, M. Holm and B.E.W. Nilsson.

"Superembeddings, non-linear supersymmetry and 5-branes", hep-th/9711203,

to appear in Int. J. Mod. Phys. A;

P.S. Howe and E. Sezgin, " $D=11, p=5$ ", Phys. Lett. B394 (1997) 62 hep-th/9611008;

P.S. Howe, E. Sezgin and P.C. West,

"Covariant field equations of the M theory five-brane", Phys. Lett. B399 (1997) 49 hep-th/9702008;

"The six-dimensional self-dual tensor", Phys. Lett. B400 (1997) 255 hep-th/9702111;

E. Bergshoeff, M. de Roo and T. Ortin, "The eleven-dimensional five-brane",

Phys. Lett. B386 (1996) 85 hep-th/9606118.

[5] M. Cederwall, B.E.W. Nilsson and P. Sundell, "An action for the 5-brane in D=11 supergravity", JHEP 04 (1998) o07 hep-th/9712059.

[6] I. Bandos, K. Lechner, A. Nurmagambetov, P. Pasti, D. Sorokin and M. Tonin, "Covariant action for the super-five-brane of M-theory",

Phys. Rev. Lett. 78 (1997) 4332 hep-th/9701037.

[7] C.G. Callan, Jr., J.A. Harvey and A. Strominger, "Worldbrane actions for string solitons", Nucl. Phys. B367 (1991) 6o.

[8] D.M. Kaplan and J. Michelson, "Zero modes for the D=11 membrane and five-brane", Phys. Rev. D53 (1996) 3474 hep-th/9510053.

[9] E. Cremmer, B. Julia and J. Scherk, "Supergravity theory in eleven-dimensions", Phys. Lett. B76 (1978) 409;

L. Brink and P. Howe, "Eleven-dimensional supergravity on the mass-shell in superspace", Phys. Lett. B91 (1980) 384;

E. Cremmer and S. Ferrara, "Formulation of eleven-dimensional supergravity in superspace", Phys. Lett. B91 (1980) 61.

[10] P.S. Howe and P.C. West, "The complete N=2, $d=10$ supergravity", Nucl. Phys. B238 (1984) 181.

[11] E. Bergshoeff, E. Sezgin and P.K. Townsend, "Supermembranes and eleven-dimensional supergravity", Phys. Lett. B189 (1987) 75;

"Properties of the eleven-dimensional supermembrane Theory", Ann. Phys. 185 (1988) 330.

[12] M.J. Duff and K.S. Stelle, "Multimembrane solutions of D=11 supergravity",

Phys. Lett. B253 (1991) 113. 
Adawi, Cederwall, Gran, Nilsson, Razaznejad: "Goldstone Tensor Modes" ......... 21

[13] R. Güven, "Black p-brane solutions of D=11 supergravity theory", Phys. Lett. B276 (1992) 49.

[14] M.J. Duff and J.X. Lu, "The self-dual type IIB superthreebrane", Phys. Lett. B273 (1991) 409.

[15] M.J. Duff, R.R. Khuri and J.X. Lu, "String solitons", Phys. Report. 259 (1995) 213 hep-th/9412184.

[16] M. Cederwall and A. Westerberg, "World-volume fields, $\mathrm{SL}(2 ; \mathbb{Z})$ and duality: the type IIB 3-brane", JHEP 02 (1998) o04 hep-th/9710007.

[17] P.S. Howe, N.D. Lambert and P.C. West, "The self-dual string soliton",

Nucl. Phys. B515 (1998) 203 hep-th/9709014;

J.P. Gauntlett, N.D. Lambert and P.C. West, "Supersymmetric fivebrane solitons", hep-th/9811024.

[18] M. Cederwall, A. von Gussich, B.E.W. Nilsson and A. Westerberg,

"The Dirichlet super-three-brane in type IIB supergravity",

Nucl. Phys. B490 (1997) 163 hep-th/9610148;

M. Cederwall, A. von Gussich, B.E.W. Nilsson, P. Sundell and A. Westerberg,

"The Dirichlet super-p-branes in type IIA and IIB supergravity",

Nucl. Phys. B490 (1997) 179 [hep-th/9611159;

M. Aganagic, C. Popescu and J.H. Schwarz, "D-brane actions with local kappa symmetry",

Phys. Lett. B393 (1997) 311 hep-th/9610249;

"Gauge-invariant and gauge-fixed D-brane actions", Nucl. Phys. B495 (1997) 99 hep-th/961208d;

E. Bergshoeff and P.K. Townsend, "Super D-branes", Nucl. Phys. B490 (1997) 145 hep-th/9611173.

[19] M. Cederwall and P.K. Townsend, "The manifestly $\operatorname{SL}(2 ; \mathbb{Z})$-covariant superstring", JHEP 09 (1997) 003 hep-th/9709002.

[20] D. Sorokin and P.K. Townsend, "M-theory superalgebra from the M-5-brane", Phys. Lett. B412 (1997) 265 hep-th/9708003.

[21] M. Cederwall, U. Gran, M. Holm and B.E.W. Nilsson, in preparation.

[22] J. Bagger and A. Galperin, "New Goldstone multiplet for partially broken supersymmetry", Phys. Rev. D55 (1997) 1091 hep-th/9608177;

"The tensor Goldstone multiplet for partially broken supersymmetry", Phys. Lett. B412 (1997) 296 hep-th/9707061. 